Meta

Journal des traducteurs

Translators' Journal

\title{
François-Xavier Garneau, traducteur
}

\section{Marc Lebel}

Volume 22, numéro 1, mars 1977

Histoire de la traduction au Canada

URI : https://id.erudit.org/iderudit/003428ar

DOI : https://doi.org/10.7202/003428ar

Aller au sommaire du numéro

Éditeur(s)

Les Presses de l'Université de Montréal

ISSN

0026-0452 (imprimé)

1492-1421 (numérique)

Découvrir la revue

Citer cet article

Lebel, M. (1977). François-Xavier Garneau, traducteur. Meta, 22(1), 33-36.

https://doi.org/10.7202/003428ar d'utilisation que vous pouvez consulter en ligne.

https://apropos.erudit.org/fr/usagers/politique-dutilisation/ 


\section{François-Xavier Garneau, traducteur}

Les biographes de François-Xavier Garneau glissent rapidement sur le fait qu'il fut de 1842 à 1844 traducteur à l'Assemblée législative du Canada-Uni. Cette expérience mérite certes plus que les quelques lignes que lui accordent tour à tour l'abbé Casgrain, P.-J.-O. Chauveau et Gustave Lanctôt. Au reste, la question en soulève aussitôt une autre, d'une portée plus générale, et qu'il faut bien poser. Quelle connaissance Garneau avait-il de l'anglais? Cette connaissance, où et comment l'avait-il acquise ? Nous nous proposons de montrer que Garneau n'est pas venu sans préparation et comme accidentellement à la traduction. Non seulement s'y était-il livré à quelques reprises auparavant, mais ses principales activités depuis le temps de sa cléricature chez le notaire Archibald Campbell (1825-1830) lui avaient procuré une appréciable maîtrise de là langue anglaise.

À coup sûr, Garneau n'apprend la langue ni à l'école qu'il fréquente brièvement, d'abord chez le «bonhomme Parent », puis chez Joseph-François Perrault, ni surtout au Petit Séminaire de Québec dont la porte lui demeure fermée. Sorti d'une famille fort modeste (ses parents sont analphabètes et le père de FrançoisXavier, véritable maître-jacques, tâtera sans succès tous les métiers), Garneau ne doit sa formation qu'à lui-même et aux fortes influences qu'il subit.

Suivant une tradition que rapporte Casgrain, Archibald Campbell ouvre sa bibliothèque au jeune clerc et lui fait découvrir les classiques anglais. Chose certaine (et ce témoignage constitue la plus lointaine indication qu'il ait laissée de sa compréhension de l'anglais), Garneau assiste aux représentations que donne à Québec en 1826 le grand comédien Edmund Kean, alors en tournée : de l'expérience il conserve une admiration qui ne se démentira jamais à l'égard de Shakespeare. Le même Campbell offre à Garneau deux ans plus tard l'occasion d'un voyage aux Etats-Unis sans doute fort rapide mais qui le marque profondément.

Mais plus important encore pour notre propos est le second voyage de Garneau, celui-là en France et en Angleterre. Ce voyage qu'il envisage assez court et principalement occupé à la découverte de Paris (les journées de juillet 1830 ont enflammé son imagination, tout comme celle d'une partie de la jeunesse canadienne), Garneau l'étirera tout près de deux ans et, chose inattendue, Londres sera le principal lieu de son séjour. 
Lorsqu'il s'embarque en juin 1831, Garneau ignore, bien entendu, les changements que subira le cours de son voyage. Aussi devons-nous attacher un prix particulier à ses lectures pendant la traversée. Dans ses malles il a mis quelques livres, «surtout des livres anglais » afin, ajoute-t-il, de se «familiariser plus que je ne l'étais avec la langue de l'un des pays que j'allais visiter». Et de nous présenter ses auteurs : les poètes Byron et Prior, qu'il cite avec ravissement, et puis Newton, «ce prince de l'astronomie ».

Une fois à Londres, et sans qu'il ait sollicité le poste, Garneau devient le secrétaire de Denis-Benjamin Viger, envoyé de l'Assemblée du Bas-Canada auprès du Colonial Office. Copie de rapports et correspondance l'occupent plus qu'il ne le désire. De ses besognes, il s'échappe tout de même : ses lettres et sa relation de voyage nous le montrent curieux des réalités anglaises. Il va au théâtre, devient membre d'une société de discussion, fréquente la bibliothèque du British Museum, visite musées, galeries et parcs. Il assiste aux séances de la Chambre des communes et se rend à des assemblées publiques, notamment à celles fort houleuses qui entourent la question de la Réforme électorale. L'éloquence qui s'y déploie le soulève, l'emporte. À Westminster Abbey, "cette cité funèbre de rois et de héros », il se recueille souvent devant les cendres d'écrivains et d'hommes politiques admirés.

Si Garneau manifeste une indéniable admiration à l'égard de la littérature et des institutions anglaises, il est moins sûr que son séjour londonien le métamorphose en un anglophile caractérisé. Trop d'obstacles l'en empêchent, et l'Angleterre ne cesse pas à ses yeux d'être l'orgueilleuse et puissante métropole d'un énorme empire colonial, l'ennemi héréditaire de la France à qui elle a arraché le Canada. Mais il nous suffit de noter que Garneau donne à Londres tous les signes d'une maîtrise fort honorable de la langue.

Ici, on doit observer que la ville de Québec, aussi bien que les livres et les voyages, favorise l'acquisition et l'approfondissement de l'anglais chez Garneau. La capitale compte un nombre important de Britanniques, les uns nouvellement arrivés, les autres établis depuis une ou deux générations. En 1819, à l'époque où grandit Garneau, ce groupe représente $27,5 \%$ de la population ; onze ans plus tard, en 1840 , il en constitue plus du tiers, soit $36,8 \%$. L'influence de ce groupe est manifeste en tous domaines. Aussi est-ce sans surprise que nous rencontrons des Britanniques parmi les pensionnaires que loge la famille Garneau entre 1828 et 1831. L'examen du greffe de François-Xavier Garneau est lui-même fort significatif : la plupart des actes mettent en cause des Britanniques et sont rédigés en anglais.

En vérité, Garneau ne pratique le notariat que par intermittence à son retour d'Europe. Il cherche à quitter une profession qui ne lui convient guère. Sans doute en mai 1834 s'associe-t-il à un confrère en vue, Louis-Théodore Besserer, membre de la Chambre d'assemblée. Mais à la lecture du contrat, on découvre que l'association, assez rapidement dissoute d'ailleurs, accorde à l'un et à l'autre des absences prolongées et que Garneau pratique une activité parallèle de traducteur. Tandis que Besserer s'absentera \& pour vaquer aux affaires de la Légis- 
lature », Garneau «pourra y aller traduire \& travailler à son profit \& avantage pendant et après la session $»$. Travaux alimentaires d'un notaire désargenté ? On a tout lieu de le croire. Il ne subsiste hélas aucune trace de ce second métier de Garneau et nous en ignorons à peu près tout, sinon qu'il semble s'agir de traductions à la pige qui lui permettent, avantage appréciable, de se rapprocher de la Bibliothèque de la Chambre d'assemblée, la seule qui vaille à Québec.

Ayant fait des adieux à peu près définitifs au notariat, Garneau devient commis de banque au printemps de 1837 et le demeure jusqu'à la fin de 1841 . Tout comme son étude de notaire, ce poste de caissier lui laisse d'amples loisirs que le poète, le journaliste, l'historien en herbe et également le traducteur savent exploiter. Au cours de ces années (1837-1841), Garneau fait preuve d'une intense activité. Non seulement le voyons-nous poursuivre une ouvre poétique, publier ses premiers travaux historiques et lancer l'Institut, toutes choses connues, mais aussi, et du point de vue qui est le nôtre ici, il paraît encore se livrer à la traduction. C'est du moins ce que donne à penser un texte qu'il adresse au Canadien en 1841 et dont l'importance tient au fait qu'il est peut-être le seul dans toute l'œuvre de traducteur de Garneau que nous pouvons lui attribuer sans l'ombre d'un doute.

A y bien regarder, l'énergie un peu brouillonne que déploie Garneau dissimule une évolution fondamentale, mais difficile à saisir. $\AA$ défaut de la correspondance (celle-ci connaît une malheureuse interruption d'avril 1835 à avril 1841) et en l'absence de toute précision ou confidence faite dans la suite, on peut seulement constater que l'intellectuel accablé par l'échec de 1837 et l'iniquité de l'Acte d'Union discipline petit à petit ses dons. Le journaliste et le poète s'effacent progressivement devant l'historien. Non plus divertissement de dilettante ou activité passagère d'historien du dimanche, l'étude du passé devient chez Garneau une passion avide et bientôt exclusive qui inspire et ordonne son existence.

C'est cette passion, semble-t-il, qui le pousse, en mai 1841, au départ de la Bibliothèque de la Chambre d'assemblée pour Kingston, à dénoncer en des termes inhabituellement vifs la « spoliation », l' \& acte de vendalisme, le * vol sacrilège » dont Québec est la victime. Privé depuis la dernière insurrection des richesses de cette bibliothèque (elle compte alors 6000 volumes), Garneau voit disparaître avec une particulière amertume les quelque $\$ 700$ volumes d'histoire, de voyages et de découvertes, tous relatifs au Canada et à l'Amérique, ramassés à grands frais [...] et qu'il sera presque impossible de remplacer $\gg$.

C'est cette même passion, croyons-nous, qui l'amène un an plus tard a prendre la route de Kingston où se réunit toujours la nouvelle Assemblée législative et à devenir assistant traducteur français.

Il est bien possible, comme le pense Gustave Lanctôt, que Garneau doive la place à l'intervention d'Étienne Parent, député et sous peu greffier du Conseil exécutif. En tout cas, cette place arrive à point nommé. Elle lui procure un traitement de $£ 200$. Garneau peut ainsi soutenir une famille qui ne cesse de s'accroître, tout en ne s'éloignant d'elle, somme toute, qu'assez peu. 
Car il faut souligner que Garneau séjourne brièvement à Kingston. Pendant les deux années où il occupe le poste, il ne s'y trouve que pour la durée de la deuxième et de la troisième sessions du premier Parlement tenues du 8 septembre au 12 octobre 1842 et du 28 septembre au 9 décembre 1843 : ce qui fait moins de 12 semaines. Sinon, Garneau demeure à Québec et y fait ses traductions qu'il achemine au traducteur en titre, Henri Voyer.

Si brefs soient-ils, les séjours de Garneau à Kingston lui permettent toutefois d'avancer son grand cuvre. Mis à portée de la Bibliothèque de l'Assemblee, il peut en exploiter les ressources sur les points particuliers où les bibliothèques de Québec réunies se révèlent insuffisantes.

Enfin, considération non négligeable, la présence de Garneau à Kingston le rapproche de personnalités susceptibles de favoriser son entreprise. C'est ainsi qu'à l'automne de 1843 il fait courir parmi les députés et les conseillers législatifs une souscription en vue de son Histoire en préparation. D'autre part, on peut penser qu'il y noue les relations qui lui faciliteront à quelques reprises dans la suite l'obtention d'une aide matérielle de l'Assemblée.

La correspondance de Garneau ne nous instruit pas sur la nature des textes que lui-même et Henri Voyer traduisent, mais il s'agit sans doute du journal de l'Assemblée législative et de ses appendices. Tâche encore légère à l'époque, mais qui va rapidement s'alourdir et appeler une réforme : au lendemain du départ de Garneau, en août 1844, le nombre des traducteurs français passe de deux à quatre, et la Chambre paraît exiger d'eux une plus grande assiduité.

Que la traduction ait été pour Garneau, en ces années décisives de recherches, de mise en place et de rédaction, une solution commode et peu accaparante à ses obligations familiales, il est à peine nécessaire de le souligner. On le sent tout entier à son œuvre, dont il publie d'ailleurs dans la presse périodique des fragments, une importante esquisse et bientôt le prospectus.

Le même calcul, le même souci de s'assurer des loisirs et une relative indépendance conduit Garneau à occuper pendant vingt ans, d'août 1844 à mai 1864, le poste de greffier de la ville de Québec. Au cours de cette dernière période de sa vie, l'admirateur de Shakespeare et de Milton continue de pratiquer la littérature anglaise ; le catalogue de sa bibliothèque personnelle et le registre du prêt de l'Institut canadien font voir une inclination particulière pour les poètes. Quant à l'historien, il achève, puis reprend inlassablement son Histoire du Canada. Et dès 1850 , il sent l'utilité d'une traduction de l'ouvrage. Celle-ci paraîtra dix ans plus tard, œuvre d'Andrew Bell, au grand mécontentement de Garneau qui la juge infidèle. Ả l'Hôtel de Ville, la familiarité de Garneau avec la langue anglaise et son expérience de traducteur lui sont précieuses, puisque la fonction l'oblige notamment à tenir dans les deux langues les procès-verbaux du Conseil.

MARC LeBel 